\title{
Effect of off-center ion substitution in morphotropic lead zirconate titanate
}

Mohan K. Bhattarai ${ }^{1}$, Shojan P. Pavunny ${ }^{1 * \dagger}$, Alvaro A. Instan ${ }^{1}$, James F. Scott ${ }^{2}$, and Ram S.

$\operatorname{Katiyar}^{1 *}$

${ }^{1}$ Department of Physics and Institute for Functional Nanomaterials, University of Puerto Rico, P.O. Box 70377, San Juan, PR 00936-8377, USA

${ }^{2}$ School of Chemistry and Physics, St. Andrews University, St. Andrews, Scotland KY16 9ST, UK

\begin{abstract}
A detailed study of the effect of off-center donor ion $\left(\mathrm{Sc}^{3+}\right)$ substitution on structural, microstructural, optical, dielectric, electrical and ferroelectric properties of morphotropic lead zirconate titanate electroceramics with the stoichiometric formula $\mathrm{Pb}_{0.85} \mathrm{Sc}_{0.10} \mathrm{Zr}_{0.53} \mathrm{Ti}_{0.47} \mathrm{O}_{3}$ (PSZT), synthesized using a high-energy solid-state reaction technique, was carried out. Powder $\mathrm{x}$-ray diffractometry was used to identify the stabilized tetragonal phase (space group $P 4 m m$ ) with considerably reduced tetragonal strain, $c / a=1.005$. An analysis of the thermal dependence of the Raman results indicated a smooth order-disorder displacive (ferroelectric-paraelectric) phase transition as revealed by the observed disappearance of the soft modes $A_{1}(1 T O)$ and $A_{1}$ (2TO) above $460 \mathrm{~K}$. The dielectric response of Pt/PSZT/Pt metal-ferroelectric-metal (MFM) capacitors was probed over a wide range of thermal excursions $(85-600 \mathrm{~K})$ and ac signal frequencies $\left(10^{2}-10^{6} \mathrm{~Hz}\right)$. Thermally activated dynamic and static conduction processes indicate hopping conduction mechanism $\left(E_{a c t} \leq 0.015 \mathrm{eV}\right.$ ) and the formation of small polarons caused by the electron and/or hole-lattice (phonon) interaction $\left(E_{a c t} \geq 0.1 \mathrm{eV}\right)$ at low $(100-300 \mathrm{~K})$ and high temperatures $(300-600 \mathrm{~K})$, respectively. The reduction in remnant polarization obtained is in good agreement with the largely reduced tetragonal strain observed in this sample, $\left(P_{r} \propto\right.$ $\sqrt{c / a-1})$. DC current conduction is dominated by Poole-Frenkel mechanism that assumes a Coulombic attraction between de-trapped electrons and positively charged stationary defects in the polycrystalline matrix.
\end{abstract}

*Corresponding authors: e-mail:shojanpp@gmail.com (S. P. P.), rkatiyar@hpcf.upr.edu (R. S. K). Tel.: 787751 4210. Fax: 7877642571.

† Present address: U.S. Naval Research Laboratory, 4555 Overlook Ave SW, Washington DC 20375. 


\section{INTRODUCTION}

Ferroelectric perovskites with the general formula $\mathrm{ABO}_{3}$ have a monovalent or divalent and a pentavalent or tetravalent cation at the A and B sites, respectively, and $\mathrm{O}$ represents the divalent oxygen anion. The simple lead zirconate titanate $\mathrm{Pb}\left(\mathrm{Zr}_{\mathrm{x}} \mathrm{Ti}_{1-\mathrm{x}}\right) \mathrm{O}_{3}(0 \leq \mathrm{x} \leq 1)$ or PZT, which is a solid solution of lead zirconate $\left(\mathrm{PbZrO}_{3}\right)$ and lead titanate $\left(\mathrm{PbTiO}_{3}\right)$, is one of the most researched and technologically important perovskite oxides due to its exceptional ferroelectric and piezoelectric properties. ${ }^{1}$ Depending on the composition PZT possesses two ferroelectric phases at room temperature: a tetragonal phase (space group $P 4 \mathrm{~mm}$ ) in the titanium-rich composition with $\mathrm{Pb}$-ion displaced along the 4-fold axis (statistically averaged - the local displacements are all [111] axes, with rapid hopping among [111], [1-11], etc.); in the zirconiumrich composition there is a rhombohedral phase (space group $R 3 c$ or $R 3 m$ ) with $\mathrm{Pb}$-ion displaced along the 3 -fold axis, that is, along the cubic [111] direction., ${ }^{2,3}$ An enhancement of the piezoelectric properties in PZT has been observed at the morphotropic phase boundary, a region of the temperature-composition phase diagram where the tetragonal to rhombohedral phase transition occurs through an intermediate monoclinic (space group $\mathrm{Cm}$ ) phase as $\mathrm{x}$ exceeds $\sim 52 .{ }^{4}$ PZT with composition ( $\mathrm{Zr} / \mathrm{Ti}:$ 53/47) exhibits extraordinarily high dielectric, pyroelectric, piezoelectric, and ferroelectric characteristics and undergoes a ferroelectric (tetragonal)paraelectric (cubic) phase transition at $\sim 387^{\circ} \mathrm{C} .{ }^{5}$

The electrophysical properties such as permittivity, ferroelectric coercitivity, electrical resistivity, Curie temperature, and crystal structure of PZT can be systematically modified by isovalent or heterovalent ion doping as well as substitution into $\mathrm{Pb}$ and/or $\mathrm{Zr} / \mathrm{Ti}$ sites, resulting in the design of new higher-performance material compositions. A-site or B-site donor (chemical valences higher than the original $\mathrm{A} / \mathrm{B}$ site ions) substitution can decrease the fatigue in PZT, 
which is attributed to the diluted oxygen vacancy concentration $\left(V_{o}^{*}\right)$ and compensated $\mathrm{Pb}$ vacancies $\left(V_{P b}^{\prime \prime}\right)$, and mediate hole concentration by the donors. Zhang et al. theoretically predicted that fatigue behavior in PZT can be improved by A-site substitution using heterovalent group IIIA elements $\left(\mathrm{La}^{3+}, \mathrm{Sc}^{3+}\right.$, or $\left.\mathrm{Y}^{3+}\right)$, which results in its conduction band minima being shared by $\mathrm{Ti} 3 \mathrm{~d}$ and impurity states, leading to a reduction in occupation in Ti $3 \mathrm{~d}$ states by the oxygen vacancies' freed electrons. ${ }^{6}$ The substitution of these elements free electrons to compensate the holes in volatile $\mathrm{Pb}$ atom sites and dilute the oxygen vacancy concentration causes the softening of the ferroelectric features of PZT, which provides new knowledge of the effects of donor substitution on the electrophysical properties of PZT, and may aid in the design of new smart/intelligent materials. ${ }^{7}$

With this motivation a systematic investigation on crystal structure, microstructure, electrical, dielectric and ferroelectric properties of A-site donor (scandium) substituted lead zirconate titanate electroceramics $\left(\mathrm{Pb}_{0.85} \mathrm{Sc}_{0.10} \mathrm{Zr}_{0.53} \mathrm{Ti}_{0.47} \mathrm{O}_{3}\right)$ in which $10 \%$ of the A-sites have $\mathrm{Sc}^{3+}$ and 5\% are vacant have been carried out. Higher dopant levels do not result in single-phase material.

\section{EXPERIMENTAL PROCEDURE}

High purity powders of lead (II) oxide (99.9\%), zirconium (IV) oxide (99.5\%), titanium (IV) oxide (99.8\%) and scandium oxide (99.9\%) from Alpha Aesar products were used to synthesize PSZT ceramics by a conventional solid state reaction method. All of these oxide powders were weighed in accordance with the stoichiometric molecular formula $\mathrm{Pb}_{0.85} \mathrm{Sc}_{0.10} \mathrm{Zr}_{0.53} \mathrm{Ti}_{0.47} \mathrm{O}_{3}$ (PSZT), mixed together, and ball milled for $24 \mathrm{hrs,} \mathrm{with} \mathrm{stabilized} \mathrm{zirconia} \mathrm{balls} \mathrm{in} \mathrm{methanol.} \mathrm{An}$ excess of 10 weight $\% \mathrm{PbO}$ was added to recover the loss of lead during calcination and sintering at high temperatures. The ball-milled mixture was subsequently dried, finely crushed using 
mortar and pestle and calcined at $1050{ }^{\circ} \mathrm{C}$ for $6 \mathrm{hrs}$ in a Carbolite HTF1700 furnace. The phase purity of powders was checked at room temperature by employing a Rigaku Ultima III X-ray diffractometer (XRD) equipped with $\mathrm{CuK} \alpha$ radiation $(\lambda=1.5405 \AA)$ source configured in BraggBrentano $(\theta-2 \theta)$ geometry and operating at $40 \mathrm{kV}$ and $40 \mathrm{~mA}$. The synthesized powders were mixed with 5 wt $\%$ polyvinyl alcohol as binding agent and were pressed in the form of thick pellets (13 mm diameter; $1 \mathrm{~mm}$ thickness) at a uniaxial pressure of $3.9 \times 10^{4} \mathrm{~Pa}$ and later sintered at $1150{ }^{\circ} \mathrm{C}$ for $5 \mathrm{hrs}$. The XRD spectra were again measured to confirm the phase purity. The samples were then subjected to Raman spectroscopy studies in the temperature range from $80 \mathrm{~K}$ to $580 \mathrm{~K}$. We used a Coherent Argon ion laser (Innova 70-C) of wavelength 514.5nm. A liquidnitrogen-cooled charge-coupled detector collected the Raman scattered signal through a $50 \times$ objective. We collected temperature dependent spectra of the sample in vacuum from $80 \mathrm{~K}$ to $580 \mathrm{~K}$ using a Linkam module. The morphology, size, shape and distribution of the samples were analyzed at room temperature in vacuum at $3500 \times, 5000 \times$ and $10000 \times$ magnifications using a scanning electron microscope (SEM) model JOEL JSM-6480LV having a resolution of better than $1 \mu \mathrm{m}$ and operating at a potential of $20 \mathrm{kV}$. Elemental analysis of the pellets was carried out by recording the energy-dispersive $\mathrm{x}$-ray (EDX) spectra. For dielectric and electrical measurements, synthesized pellets were DC sputtered at room temperature with platinum and were subsequently annealed at $400^{\circ} \mathrm{C}$ for $30 \mathrm{~min}$ in a tube furnace at ambient environment for proper adhesion of $\mathrm{Pt}$ and to compensate for any sputter damage. The capacitance versus frequency $(\mathrm{CF})$, capacitance versus voltage $(\mathrm{CV})$ and loss tangent were measured using an HP4294A Impedance Analyzer at a low ac test signal of $250 \mathrm{mV}$, and current versus dc voltage (IV) measurement were carried out with a Keithley electrometer (model \#6517A) in the temperature range of $85 \mathrm{~K}$ to $600 \mathrm{~K}$. Temperature control was achieved using a programmable 
temperature controller (MMR technologies, Inc.). The samples were poled for 7 hours, and the remnant polarization and coercive field of the ferroelectric capacitors were measured using a Sawyer Tower test configuration (Radiant Technologies Model RT 6000 HVA-4000V).

\section{RESULTS AND DISCUSSION}

\section{A. Structural and microstructural analysis}

The new phase diagram of PZT reported by Zhang et al. predicts the coexistence of tetragonal (space group $P 4 m m$ ), rhombohedral (space group $R 3 m$ ) and monoclinic (space group $\mathrm{Cm}$ ) room temperature structures at the morphotropic phase boundary. ${ }^{8}$ The coexistence of tetragonal (space group $P 4 \mathrm{~mm}$ ) and monoclinic (spacegroup $\mathrm{Cm}$ ) phases as a result of a first order phase transition between the low temperature monoclinic and high temperature tetragonal phases were identified from x-ray diffraction refinement of pure $\mathrm{PbZr}_{\mathrm{x}} \mathrm{Ti}_{1-\mathrm{x}} \mathrm{O}_{3}$ ceramics $(0.515<\mathrm{x}<0.530) .{ }^{9}{ }^{910}$ A ferroelectric $\mathrm{PbZr}_{0.52} \mathrm{Ti}_{0.48} \mathrm{O}_{3}$ composition with tetragonal crystal structure and $P 4 m m\left(C_{4 v}^{1}\right)$ space group has been reported by several researchers. ${ }^{5,11}$ The room temperature XRD diffraction pattern of PSZT ceramics collected at a slow angular velocity of $0.3 \mathrm{deg} / \mathrm{min}$ shown in Fig. 1(a) suggest that the material possesses a homogenous tetragonal crystal structure. For cubic symmetry with lattice parameters $a=b=c$ splitting of (200), (220), (222) Bragg peaks are not expected. For the tetragonal structure, the (200) peak is expected to be a doublet and (222) a singlet. However, the (222) x-ray peak for the rhombohedral phase should exhibit a doublet, whereas for monoclinic it is a quadruplet. In our case (200), (220) and (222) peaks were more accurately Gaussian modeled with doublet, doublet and singlet, respectively (Fig. 1insets (a-c)), which shows that PSZT ceramics possess a stabilized tetragonal symmetry with space group $P 4 m m$ which does not have any inversion center. Additionally, the Goldschmidt tolerance factor, ${ }^{12} 0.8319$, estimated for PSZT using octahedral radii of $\mathrm{Pb}^{2+}(1.29$ 
$\AA), \mathrm{Sc}^{3+}(0.87 \AA), \mathrm{Zr}^{4+}(0.84 \AA), \mathrm{Ti}^{4+}(0.74 \AA)$ and $\mathrm{O}^{2-}(1.42 \AA)^{13}$ favors its tetragonal stable phase formation with higher symmetry (decreased degree of distortion). We observed a shift in Bragg peaks towards higher diffraction angle with respect to those of undoped $\mathrm{PbZr}_{0.53} \mathrm{Ti}_{0.47} \mathrm{O}_{3}$ (data not shown) as a result of decrease in lattice constants due to the incorporation of soft/donor dopant $\mathrm{Sc}^{3+}$ (ionic radius $0.87 \AA$ ) at the $\mathrm{Pb}^{2+}$ (ionic radius $1.29 \AA$ ) site. The room temperature lattice parameters of PSZT were estimated to be $a=b=4.07 \AA$ and $c=4.09 \AA$ (unit cell volume $=67.78 \AA^{3}$ ) using Rietveld structural analysis assuming the tetragonal structure with $P 4 m m$ space group (data not shown). The greatly reduced tetragonal strain, $c / a=1.005$, is a clear evidence of Sc incorporation in the PZT matrix. Figure 2 depicts EDX spectrum of the PSZT sample excited by an electron beam of energy $20 \mathrm{kV}$, showing the presence of elements $(\mathrm{Pb}, \mathrm{Zr}, \mathrm{Ti}, \mathrm{Sc}$ and $\mathrm{O})$ making up the oxide compound, along with their respective characteristic x-ray emission lines (O Ka 0.525 keV, Zr L $\alpha 2.042$ keV, Sc Ka 4.089 keV, Ti Ka 4.508 keV, Pb $\mathrm{M} \beta 5.076 \mathrm{keV}$ and $\mathrm{Pb} \mathrm{L} \alpha 10.552 \mathrm{keV})$. On average, the $\mathrm{Pb}: \mathrm{Sc}: \mathrm{Zr}: \mathrm{Ti}$ atomic ratio, estimated from the intensities of the respective characteristic lines, was in good agreement with the composition stoichiometry of PSZT precursors prior to calcination. The inset of Fig. 2 depicts a typical SEM micrograph of the sintered pellet revealing pores and well defined granular structure consisting of randomly oriented grains (crystallites) with an average grain size of about $\sim 1-2 \mu \mathrm{m}$.

\section{B. Raman spectroscopic studies}

Raman vibrations of ferroelectric tetragonal phase with one formula unit in the primitive cell belong to point group $4 m m$ and space group $P 4 m m$ or $C_{4 v}^{1}$; its normal modes are given by: , $\Gamma_{\text {opt }}=3 A_{1}(r, i r)+4 E(r, i r)+B_{1}(r)$ where $\Gamma_{\text {opt }}$ are the optical vibrational modes and the symbols $r$ and $i r$ indicate Raman- and infrared-active modes, respectively. ${ }^{14,15}$ Each of the three $A_{1}$ and three $E$ modes is doubly degenerate with a transverse mode (TO) having polarization 
perpendicular to propagation $\vec{k}$ and a longitudinal mode (LO) with polarization parallel to $\vec{k}$ due to long-range Coulomb forces, making the total number of spectral peaks 14. Raman spectra of PSZT polycrystalline samples recorded in the temperature window of $80-580 \mathrm{~K}$ are presented in Fig. 3. The observed overlap in spectra and their large line-widths that broadened with temperature makes the identification of individual modes clumsy. It can be caused by the static atomic displacement from their ideal positions as a result of lattice strain due to the material's thermal history as well as due to compositional substitution by atoms with mismatched cationic radii and valencies. ${ }^{16}$ In our case the $\mathrm{Sc}^{3+}$ substitution resulted in a nearly cubic structure with highly reduced tetragonality, as revealed from x-ray diffractometry studies. Nevertheless, five modes $\mathrm{E}(1 \mathrm{LO}), \mathrm{A}_{1}(1 \mathrm{TO}), \mathrm{E}(2 \mathrm{TO}), \mathrm{E}+\mathrm{B} 1$, and $\mathrm{A}_{1}(2 \mathrm{TO})$ were identified with certainty and the peak frequency of TO modes was found to be reduced by the substitution of $\mathrm{Pb}$ (atomic weight 207.2) sites by Sc (atomic weight 44.96) as a result of appreciable softening of force constant of the respective lattice vibration together with a large reduction in ferroelectric distortion or $c / a$ ratio. The presence of prominent $A_{1}(1 \mathrm{TO})$ and $A_{1}(2 \mathrm{TO})$ modes in the $80-460 \mathrm{~K}$ temperature window show that the tetragonal phase is intact in this range. The soft mode $A_{1}(1 T O)$, which originates from the vibrations of $\mathrm{Ti} / \mathrm{Zr}$ and $\mathrm{O}$ ions relative to $\mathrm{Pb} / \mathrm{Sc}$ ions, and $\mathrm{A}_{1}$ (2TO), which represents the displacements of the $\mathrm{Ti} / \mathrm{Zr}$ ion with respect to both $\mathrm{O}$ and $\mathrm{Pb} / \mathrm{Sc}$ ions, are closely affected by the existence of the ferroelectric phase. ${ }^{17,18,15}$ All of the identified modes except $\mathrm{E}+\mathrm{B}_{1}$ were found to soften with the rise in temperature. ${ }^{19}$ Above $460 \mathrm{~K}$ all the modes undergo significant damping, and $A_{1}(1 \mathrm{TO})$ and $A_{1}$ (2TO) soft modes lose their intensities and disappear,which can be considered as arising from a displacive phase transition in which the ferroelectric tetragonal to paraelectric cubic symmetry distortion commences at temperatures below the disappearance of ferroelectric ordering. So the Sc substitution results in the significant 
lowering of Curie temperature $T_{C}$ of pure PZT, which is reported to be $\sim 653 \mathrm{~K}^{4}$ Only some broad and weak phonon modes were present in the spectra above $460 \mathrm{~K}$; they correspond to $\mathrm{TO}_{2}$, $\mathrm{TO}_{3}$, and $\mathrm{TO}_{4}$ hard modes (see Fig. 3) belonging to the cubic phase with $P m \overline{3} m$ or $O_{h}^{1}$ space symmetry. ${ }^{20}$ These three modes observed out of the four predicted (including $\mathrm{TO}_{1}$ ) originate in the three $\left(\mathrm{TO}_{1}, \mathrm{TO}_{2}\right.$, and $\left.\mathrm{TO}_{4}\right)$ optical odd-parity/polar $T_{1 u}$ and one $\left(\mathrm{TO}_{3}\right)$ non-polar $T_{2 u}$ modes and can be attributed to the presence of polar nanoregions at higher temperatures (below and above $\left.T_{C}\right)$ induced by off-center ion $\left(\mathrm{Sc}^{3+}\right.$ whose ionic radii is only $67 \%$ that of $\left.\mathrm{Pb}^{3+}\right)$.

\section{Diffuse Reflectance Spectroscopy}

An optical bandgap $E_{g}$ was estimated for the PSZT sample as shown in Fig. 4 using diffuse reflectance spectra under the assumption of a direct band-to-band transition between $\mathrm{O} 2 p$ valence band maximum and Ti3d and dopant Sc3d mixed conduction band minimum. The vertical line in the first derivative of Kebulka-Munk function with respect to wavelength $(\mathrm{dF} / \mathrm{d} \lambda)$ versus wavelength $(\lambda)$, given in the inset of the graph, indicates the inflection point, which represents the bandgap of the material at $\sim 363 \mathrm{~nm}(\sim 3.42 \mathrm{eV})$. This value is lower than the bandgap $\sim 3.55 \mathrm{eV}$ reported for undoped PZT (50/50) composition in thin film form. ${ }^{21}$ The observed reduction in bandgap with respect to pure PZT is in good agreement with the theoretical prediction by Zhang $e t a l .{ }^{6}$ where the phenomenon was ascribed to the formation of a mid-gap impurity band in PSZT by $\mathrm{Sc}^{3+}$ dopants.

\section{Dielectric properties}

Figure 5 depicts in a semi-logarithmic scale the variation in the real part of dielectric permittivity $\left(\varepsilon^{\prime}\right)$ and the loss tangent ( $\tan \delta$ ) of PSZT ceramic as a function of frequency (100 $\mathrm{Hz}$ to $1 \mathrm{MHz}$ ) in the temperature range of $85 \mathrm{~K}$ to $600 \mathrm{~K}$. The room-temperature dielectric 
constant and the dielectric loss were found to be $\sim 220$ (high) and $\sim 0.02$ (low), respectively, at a frequency of $100 \mathrm{kHz}$. The dielectric constant obtained was lower than the value reported for the pure PZT (53/47) sintered ceramic (about 2000). ${ }^{22}$ The dielectric constant $(240$ at $100 \mathrm{~Hz}$ and 210 at $\left.10^{6} \mathrm{~Hz}\right)$ and dielectric loss $\left(0.02\right.$ at $100 \mathrm{~Hz}$ and 0.001 at $\left.10^{6} \mathrm{~Hz}\right)$ were found to be very weakly decreasing with increasing frequency. Below $1 \mathrm{kHz}$ contribution of multi-component of polarization such as deformational polarization (electronic and ionic) and relaxation polarization (orientation and interfacial) causes the slight increase of $\varepsilon^{\prime}$. At high frequencies dipoles cannot keep up with the field and hence there is no contribution of orientation polarization, resulting in the decrease of $\varepsilon^{\prime}$ approaching a constant value due to only the interfacial polarization.

Figure 6 exhibits the variation in dielectric constant and loss tangent (inset) with temperature in the frequency range of $10^{2} \mathrm{~Hz}$ to $10^{6} \mathrm{~Hz}$. The observed frequency-independent dielectric constant increases slowly from $85 \mathrm{~K}$ to $400 \mathrm{~K}$. Above $400 \mathrm{~K}$ we observed a sharp increase in dielectric constant up to the experimental limit $600 \mathrm{~K}$.

\section{E. AC and DC conductivities}

The frequency dependence of ac conductivity $\left(\sigma_{a c}\right)$ at various temperatures $(100-600 \mathrm{~K})$ of PSZT ceramics is shown in Fig. 7. It is clear from this figure that above a certain frequency $\left(10^{4}\right.$ $\mathrm{Hz}), \sigma_{a c}$ increases linearly with frequency. Dynamic conductivity shows the typical features of Joncher's universal dynamic response (UDR) and obeys a power law: ${ }^{23}$

$$
\sigma_{a c}=\sigma_{d c}+A \omega^{n}
$$

where $\sigma_{d c}$ is the frequency independent dc conductivity; $\omega=2 \pi \mathrm{f}$, the angular frequency;

$A$, a pre-exponential factor; and $n(0<n \leq 1)$, the frequency exponent. From this plot it is evident that the DC contribution to conductivity is significant at low frequencies and high temperatures, whereas the frequency dependent term dominates conductivity at high frequencies. 
In our case the numerical values of $\sigma_{d c}$ and $n$ were estimated by modeling the experimental data with Eqn. (1), and the results are summarized in Table I. $\sigma_{d c}$ was found to increase and $n$ was found to decrease with the increase in temperature. The frequency exponent falls in the range of $0<n \leq 1$, approaches unity at low temperatures $(\leq 300 \mathrm{~K})$, and the transport is dominated by hopping in finite clusters (correlated barrier hopping).

The thermally activated conduction process at a specific frequency is governed by the Arrhenius relation:

$$
\sigma(T)=\sigma_{0} \exp -\left(E_{a c t} / k_{B} T\right)
$$

where $\sigma_{0}$ is the pre-exponential factor; $\mathrm{E}_{\text {act }}$, the activation energy; $k_{B}$, the Boltzmann constant; and $T$, absolute temperature. Figure 8 , which is divided into two temperature regions $(\mathrm{I}=100-300 \mathrm{~K}$ and $\mathrm{II}=300-600 \mathrm{~K})$, depicts the thermal behavior of $\mathrm{AC}\left(10^{2}-10^{6} \mathrm{~Hz}\right)$ and DC conductivities. The numerical values of $E_{a c t}$ obtained by least square fitting the conductivity data with Eq. 2 in the regions I and II are given in Table II. The low activation energies determined in the $100-300 \mathrm{~K}$ range, $\leq 0.015 \mathrm{eV}$, represent an n-type hopping conduction mechanism with $\mathrm{Sc}$ cations acting as donor centers. ${ }^{24}$ The increase in $E_{a c t}$ to $\geq 0.1 \mathrm{eV}$ in region II indicates the formation of small polarons induced by the electron and/or hole-lattice (phonon) interaction enhanced by structural deformations involved in the transition from ferroelectric to paraelectric phase.

\section{F. Ferroelectric properties}

Room temperature polarization versus electric field (P-E) hysteresis loops for PSZT ceramics were measured at various electric fields after poling at a high dc voltage of $1 \mathrm{kV}$ (close to the coercive field to switch the ferroelectric domains) and are shown in Fig. 9. The remnant 
polarization $\left(P_{r}\right)$ and coercive field $\left(E_{c}\right)$ of the ferroelectric capacitors recorded at $50 \mathrm{~Hz}$ using a computer-controlled Sawyer Tower test circuit were $\sim 3 \mu \mathrm{C} / \mathrm{cm}^{2}$ and $\sim 13 \mathrm{kV} / \mathrm{cm}$, respectively. The sintered $\mathrm{PbZr}_{0.52} \mathrm{Ti}_{0.48} \mathrm{O}_{3}$ ceramics prepared by sol-gel process exhibited a remnant polarization of $20 \mu \mathrm{C} / \mathrm{cm}^{2}$ and a coercive field of $10.6 \mathrm{kV} / \mathrm{cm} .{ }^{25}$ So it can be understood that doping of PZT with 10 mole $\% \mathrm{Sc}^{3+}$ results in a drastic reduction of $P_{r}$ without any marked change in $E_{c}$ and non-saturation of the $\mathrm{PE}$ loop in the experimental field range. The low ferroelectricity achieved is in good agreement with the largely reduced tetragonal strain observed in this sample as given by the relation spontaneous/remnant polarization $P_{r} \propto \sqrt{c / a-1} .{ }^{14}$

\section{E. DC leakage characteristics}

The temperature dependent $(300-600 \mathrm{~K})$ experimental J-E characteristics obtained for the $\mathrm{Pt} / \mathrm{PSZT} / \mathrm{Pt}$ MFM capacitor structure while sweeping the dc bias voltage from $-1000 \mathrm{~V}$ to +1000 $\mathrm{V}$ are presented in Fig.10. Major observations that can be made from the leakage characteristics are: a) the bottom electrode injection (+Ve voltage on top $\mathrm{Pt})$ and top electrode injection $(-\mathrm{Ve}$ voltage on top Pt) branches seem to be symmetric; the top and bottom electrodes are identical, resulting in similar potential barrier heights; b) at $300 \mathrm{~K}$ a low leakage current density of $1.35 \times$ $10^{-8} \mathrm{~A} / \mathrm{cm}^{2}$ was observed at an electric field of $12 \mathrm{kV} / \mathrm{cm}$, which validates the charge storage capabilities of the sample; c) both positive and negative I-V branches show temperature dependence with three orders of increase in leakage current with temperature increase from 300

to $600 \mathrm{~K}$. These observations hint towards Schottky emission (SE) or Poole-Frenkel (PF) as the charge transport mechanism in Pt/PSZT/Pt capacitors. The field dependent conduction mechanism is formulated as ${ }^{16}$

$$
\sigma=\sigma_{0} \exp \left(\beta E^{1 / 2} / k_{B} T\right), \quad \beta=\left(e^{3} / \alpha \pi \varepsilon_{0} \varepsilon_{\infty}\right)^{1 / 2},
$$


where $\sigma_{0}$ is the low-field conductivity of the system; E, the electric field strength in the ferroelectric; $T$, absolute temperature; $e$, the unit of electronic charge; $\varepsilon_{0}$, the permittivity of free space; $\varepsilon_{\infty}$, the electronic dielectric constant; and the coefficient $\alpha$ is 1 for PF and 4 for SE. The field dependency of current studied by plotting $E^{1 / 2} / k_{B} T$ vs $L n \sigma$ can be approximated to a straight line in the $2-12 \mathrm{kV} / \mathrm{cm}$ field range, as shown in the inset of Fig. 10. The numerical value of the optical dielectric constant, $\varepsilon_{\infty}$ (and its long wavelength refractive index, $n_{\infty}$ ) estimated from the slope of the plot [Eq. (3)] at 300K is 5.47 (2.29) and 1.31(1.14) for PF and SE, respectively. In our sample PF can be considered as the dominant conduction mechanism as the numerical value of $\varepsilon_{\infty}$ or $n_{\infty}$ obtained in this case is in fairly good agreement with the figures reported for pure PZT from optical analysis ${ }^{26,27}$ and show that SE modeling is unphysical. The PF model explicitly assumes a Coulombic attraction between a de-trapped electron and a positively charged stationary defect. The hypothesis of lead vacancies in PZT as a possible center for trapping holes has been proposed in literature, ${ }^{28}$ and these centers support the observed PF conduction mechanism.

\section{CONCLUSIONS}

Off-center ion $\left(\mathrm{Sc}^{3+}\right)$ substituted morphotropic composition PZT electroceramics were synthesized by a conventional solid-state reaction technique and the influence of donor substitution on structural, microstructural, optical, dielectric, electrical and ferroelectric properties of it is reported. A stabilized tetragonal phase (space group $P 4 m m$ ) with considerably reduced tetragonality, $c / a=1.005$ was identified by powder $\mathrm{x}$-ray diffractometry. The smooth displacive (ferroelectric-paraelectric) phase transition was revealed by Raman studies from the observed disappearance of the soft modes $\mathrm{A}_{1}(1 \mathrm{TO})$ and $\mathrm{A}_{1}$ (2TO) and the emergence of $\mathrm{TO}_{2}$, 
$\mathrm{TO}_{3}$, and $\mathrm{TO}_{4}$ hard modes above $460 \mathrm{~K}$. The dielectric response of Pt/PSZT/Pt capacitors was probed as a function of temperature $(85-600 \mathrm{~K})$ and frequency $\left(10^{2}-10^{6} \mathrm{~Hz}\right)$. Frequency dependent dynamic conductivity showed that the transport is dominated by hopping in finite clusters (UDR) and obeyed a power law $\sigma_{a c}=\sigma_{d c}+A \omega^{n}$. Thermally activated dynamic and static conductions followed an Arrhenius relation with activation energies of $\leq 0.015 \mathrm{eV}$ and $\geq$ $0.1 \mathrm{eV}$ at low $(100-300 \mathrm{~K})$ and high $(300-600 \mathrm{~K})$ temperatures. The reduced ferroelectricity measured is in accordance with the greatly reduced tetragonal strain observed in this sample. DC leakage analysis revealed that the charge transport is dominated by Poole-Frenkel mechanism that assumes a Coulombic attraction between de-trapped electrons and positively charged stationary defect species in the polycrystalline sample.

\section{ACKNOWLEDGMENTS}

This research was supported by the DOD Grant No. FA9550-16-1-0295. SPP gratefully acknowledges the financial assistance from IFN-NSF under the Grant No. EPS-1002410.

\section{References}

1 Y. Xu, "Front Matter;" in Ferroelectr. Mater. their Appl. North Holland, Amsterdam, 1991.

2 M.C. Rodríguez-Aranda, F. Calderón-Piñar, F.J. Espinoza-Beltrán, F.J. Flores-Ruiz, E. León-Sarabia, R. Mayén-Mondragón, and J.M. Yáñez-Limón, "Ferroelectric hysteresis and improved fatigue of PZT (53/47) films fabricated by a simplified sol-gel acetic-acid route," J. Mater. Sci. Mater. Electron., 25 [11] 4806-4813 (2014).

$3 \quad$ M.R. Soares, A.M.R. Senos, and P.Q. Mantas, "Phase coexistence region and dielectric properties of PZT ceramics," J. Eur. Ceram. Soc., 20 [3] 321-334 (2000).

$4 \quad$ B. Jaffe, W.R. Cook, and H.L. Jaffe, Piezoelectric ceramics. Academic Press, 1971.

5 B. Noheda, J.A. Gonzalo, L.E. Cross, R. Guo, S.-E. Park, D.E. Cox, and G. Shirane, "Tetragonal-to-monoclinic phase transition in a ferroelectric perovskite: The structure of PbZr 0.52 Ti 0.48 O 3," Phys. Rev. B, 61 [13] 8687-8695 (2000). 
Z. Zhang, P. Wu, K. Ong, L. Lu, and C. Shu, "Electronic properties of A-site substituted lead zirconate titanate: Density functional calculations," Phys. Rev. B, 76 1-6 (2007). W.X. CHENG, A.L. DING, P. QIU, X. HE, and X. ZHENG, "IMPRINT PROPERTIES OF YTTRIUM DOPED PZT THIN FILM," Integr. Ferroelectr., 75 [1] 173-179 (2005).

N. Zhang, H. Yokota, M. Glazer, Z. Ren, D. Keen, D.S. Keeble, P. Thomas, and Z.-G. Ye, "The missing boundary in the phase diagram of PbZr1-xTixO3.," Nat. Commun., 5 [May] 5231 (2014).

Ragini, R. Ranjan, S.K. Mishra, and D. Pandey, "Room temperature structure of $\mathrm{Pb}(\mathrm{Zr}$ xTi 1-xO 3) around the morphotropic phase boundary region: A Rietveld study," J. Appl. Phys., 92 [6] 3266-3274 (2002).

E.B. Araújo, E.C. Lima, J.D.S. Guerra, A.O. dos Santos, L.P. Cardoso, and M.U. Kleinke, "Evidence for the monoclinic-tetragonal phase coexistence in $\mathrm{Pb}(\mathrm{Zr} 0.53 \mathrm{Ti} 0.47) \mathrm{O} 3$ thin films," J. Phys. Condens. Matter, 20 [41] 415203 (2008).

S.K. Mishra, A.P. Singh, and D. Pandey, "Thermodynamic nature of phase transitions in $\mathrm{Pb}\left(\mathrm{Zr}_{x} \mathrm{Ti}_{1-x}\right) \mathrm{O}_{3}$ ceramics near the morphotropic phase boundary. I. Structural studies," Philos. Mag. Part B, 76 [2] 213-226 (1997).

2 V.M. Goldschmidt, "Die Gesetze der Krystallochemie," Naturwissenschaften, 14 [21] 477-485 (1926).

3 R.D. Shannon, "Revised effective ionic radii and systematic studies of interatomic distances in halides and chalcogenides," Acta Crystallogr. Sect. A, 32 [5] 751-767 (1976). G. Burns and B.A. Scott, "Lattice modes in ferroelectric perovskites: PbTiO3," Phys. Rev. B, 7 [7] 3088-3101 (1973).

5 J.D. Freire and R.S. Katiyar, "Lattice dynamics of crystals with tetragonal $<$ math display='inline' $><$ mrow $><$ msub $><$ mrow $><$ mi mathvariant $=$ 'normal' $>$ BaTiO $</$ mi $></$ mrow $><$ mrow $><$ mn $>3</$ mn $></$ mrow $></$ msub $></$ m row $><$ /math $>$ structure," Phys. Rev. B, 37 [4] 2074-2085 (1988).

6 S.P. Pavunny, Y. Sharma, S. Kooriyattil, S. Dugu, R.K. Katiyar, J.F. Scott, and R.S. Katiyar, "Holmium hafnate: An emerging electronic device material," Appl. Phys. Lett., 106 [11] (2015).

17 J. Frantti, V. Lantto, and J. Lappalainen, "Symmetry consideration of Raman modes in Nd-doped lead zirconate titanate thin films for structure characterization," J. Appl. Phys., 
79 [2] 1065 (1996).

M. Deluca, H. Fukumura, N. Tonari, C. Capiani, N. Hasuike, K. Kisoda, C. Galassi, and H. Harima, "Raman spectroscopic study of phase transitions in undoped morphotropic PbZr1-xTixO3," J. Raman Spectrosc., 42 [3] 488-495 (2011).

J. Frantti and V. Lantto, "Raman studies between 11 and $300 \mathrm{~K}$ of the effects of $\mathrm{Nd}$ additive in ferroelectric lead-titanate ceramics.," Phys. Rev. B. Condens. Matter, 54 [17] 12139-12150 (1996).

P. DiAntonio, B.E. Vugmeister, J. Toulouse, and L.A. Boatner, "Polar fluctuations and first-order Raman scattering in highly polarizable KTaO3 crystals with off-center Li and Nb ions," Phys. Rev. B, 47 [10] 5629-5637 (1993).

S. Yang, D. Mo, and X. Tang, "Determination of the Optical Properties of Sol-GelDerived Pb(Zr x Ti $(1-x)$ )O 3 Thin Films by Spectroscopic Ellipsometry," Ferroelectrics, 287 [1] 35-46 (2003). W.C. Las, P.D. Spagnol, M.A. Zaghete, and M. Cilense, "Electrical characterization of lead zirconate titanate prepared by organic solution route," Ceram. Int., 27 [4] 367-372 (2001). A.K. Jonscher, Dielectric relaxation in solids. Chelsea Dielectrics Press, London, 1983. J. Portelles, N.S. Almodovar, J. Fuentes, O. Raymond, J. Heiras, and J.M. Siqueiros, "ac conductivity in $\mathrm{Gd}$ doped $\mathrm{Pb}(\mathrm{Zr}[\mathrm{sub} 0.53$ ] Ti[sub 0.47])O[sub 3] ceramics," J. Appl. Phys., 104 [7] 073511 (2008).

A. Wu, P.M. Vilarinho, I.M.M. Salvado, and J.L. Baptista, "Sol-Gel Preparation of Lead Zirconate Titanate Powders and Ceramics: Effect of Alkoxide Stabilizers and Lead Precursors," J. Am. Ceram. Soc., 83 [6] 1379-1385 (2000). M.P. Moret, M.A.C. Devillers, K. Worhoff, and P.K. Larsen, "Optical properties of PbTiO 3, PbZr xTi 1-xO 3, PbZrO 3 films deposited by metalorganic chemical vapor on SrTiO 3," J. Appl. Phys., 92 [1] 468-474 (2002).

Z. Huang, X. Meng, P. Yang, Z. Zhang, and J. Chu, “Optical properties of PbZr[sub $\mathrm{x}] \mathrm{Ti}[$ sub 1-x]O[sub 3] on platinized silicon by infrared spectroscopic ellipsometry," Appl. Phys. Lett., 76 [26] 3980 (2000).

D. Dimos, H.N. Al-Shareef, W.L. Warren, and B.A. Tuttle, "Photoinduced changes in the fatigue behavior of SrBi2Ta2O9 and $\mathrm{Pb}(\mathrm{Zr}, \mathrm{Ti}) \mathrm{O} 3$ thin films," J. Appl. Phys., 80 [3] 1682 
(1996).

\section{Figure captions:}

FIG. 1. Room temperature XRD Bragg peaks of PSZT ceramics recorded at a slow scan rate of

$0.3 \mathrm{deg} / \mathrm{min}$. Insets (a), (b), and (c) show the (200), (220) and (222) x-ray peaks with the experimental data represented by circles, fitted data represented by solid line, and the Gaussian deconvolution represented by dashed line.

FIG. 2. EDX spectrum of PSZT polycrystalline sample measured at room temperature. The SEM micrograph of the sample captured at $10000 \times$ magnification is given in the inset. 
FIG. 3. Temperature dependent Raman spectra of PSZT ceramics in the range of $80-580 \mathrm{~K}$.

FIG. 4. Diffuse-reflectance spectra of PSZT target.

FIG. 5. (a) and (b) Variation of dielectric constant $\left(\varepsilon^{\prime}\right)$ and dissipation factor ( $\tan \delta$ ) of PSZT ceramics as a function of frequency at different temperatures.

FIG. 6. Temperature dependence of dielectric constant $\left(\varepsilon^{\prime}\right)$ and loss tangent $(\tan \delta$ ) (inset) of PSZT ceramics at various frequencies.

FIG. 7. AC conductivity $\left(\sigma_{\mathrm{ac}}\right)$ of PSZT polycrystalline ceramics as a function of frequency at several temperatures from $100 \mathrm{~K}$ to $600 \mathrm{~K}$.

FIG. 8. Electrical conductivity of PSZT ceramics as a function of reciprocal temperature.

FIG. 9. P-E hysteresis loop for PSZT ceramics at various fields.

FIG. 10. Temperature dependence of leakage current density of Pt/ PSZT/Pt MFM capacitor.

The dc current-voltage analysis depicted in the inset suggests that, it obeys the bulk limited Poole-Frenkel charge transport at room temperature.

\section{List of Tables:}

TABLE I. Power law parameters evaluated by fitting experimental data.

\begin{tabular}{|c|c|c|}
\hline Temp. $(\mathrm{K})$ & $\sigma_{d c}(\Omega \mathrm{m})^{-1}$ & $n$ \\
\hline 100 & $3.28 \times 10^{-6}$ & 0.983 \\
\hline 200 & $2.11 \times 10^{-6}$ & 0.985 \\
\hline 300 & $3.01 \times 10^{-6}$ & 0.984 \\
\hline 400 & $2.80 \times 10^{-5}$ & 0.915 \\
\hline 500 & $4.31 \times 10^{-4}$ & 0.842 \\
\hline 600 & $1.08 \times 10^{-3}$ & 0.801 \\
\hline
\end{tabular}


TABLE II. Activation energy values for AC and DC conduction processes deduced assuming Arrhenius behavior in the low $(100-300 \mathrm{~K})$ and high $(300-600 \mathrm{~K})$ temperature regions.

\begin{tabular}{|c|c|c|}
\hline \multirow{2}{*}{$\begin{array}{c}\text { Frequency } \\
(\mathrm{Hz})\end{array}$} & \multicolumn{2}{|c|}{ Activation energy $(\mathrm{eV})$} \\
\cline { 2 - 3 } & $100-300 \mathrm{~K}$ & $300-600 \mathrm{~K}$ \\
\hline $0(\mathrm{DC})$ & 0.006 & 0.15 \\
\hline $10^{2}$ & 0.015 & 0.24 \\
\hline $10^{3}$ & 0.01 & 0.18 \\
\hline $10^{4}$ & 0.006 & 0.12 \\
\hline $10^{5}$ & 0.007 & 0.10 \\
\hline $10^{6}$ & 0.004 & 0.11 \\
\hline
\end{tabular}

